\title{
Nanoscale organization of Nicastrin, the substrate receptor of the $\gamma$-secretase complex, as independent molecular domains
}

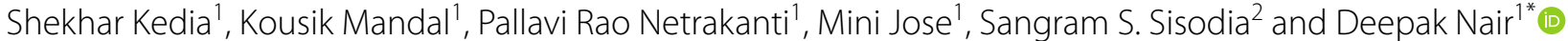

\begin{abstract}
Alterations in the canonical processing of Amyloid Precursor Protein generate proteoforms that contribute to the onset of Alzheimer's Disease. Modified composition of $\gamma$-secretase or mutations in its subunits has been directly linked to altered generation of Amyloid beta. Despite biochemical evidence about the role of $\gamma$-secretase in the generation of APP, the molecular origin of how spatial heterogeneity in the generation of proteoforms arises is not well understood. Here, we evaluated the localization of Nicastrin, a $y$-secretase subunit, at nanometer sized functional zones of the synapse. With the help of super resolution microscopy, we confirm that Nicastrin is organized into nanodomains of high molecular density within an excitatory synapse. A similar nanoorganization was also observed for APP and the catalytic subunit of $y$-secretase, Presenilin 1, that were discretely associated with Nicastrin nanodomains. Though Nicastrin is a functional subunit of $\gamma$-secretase, the Nicastrin and Presenilin 1 nanodomains were either colocalized or localized independent of each other. The Nicastrin and Presenilin domains highlight a potential independent regulation of these molecules different from their canonical secretase function. The collisions between secretases and substrate molecules decide the probability and rate of product formation for transmembrane proteolysis. Our observations of secretase nanodomains indicate a spatial difference in the confinement of substrate and secretases, affecting the local probability of product formation by increasing their molecular availability, resulting in differential generation of proteoforms even within single synapses.
\end{abstract}

Keywords: Nicastrin, Synapse, Alzheimer's disease, Presenilin, APP, Super resolution microscopy, STED, Secretase

\section{Background}

$\gamma$-secretases are the multimeric enzyme complexes consisting of four subunits, namely, Presenilin 1 (PS1), Nicastrin (NCT), Anterior Pharynx Defective 1 (APH1) and Presenilin Enhancer 2 (PEN2) [1]. NCT is a type 1 transmembrane glycoprotein and is the largest subunit of the $\gamma$-secretase enzyme complex. It is essential for the cleavage of several $\gamma$-secretase substrate molecules, importantly for Notch receptors and Amyloid Precursor

\footnotetext{
*Correspondence: deepak@iisc.ac.in

${ }^{1}$ Centre for Neuroscience, Indian Institute of Science, 560012 Bangalore, India

Full list of author information is available at the end of the article
}

Protein (APP) $[1,2]$. NCT is necessary for the final processing of APP through either the non-amyloidogenic or the amyloidogenic pathway [3]. The APP processing by $\gamma$-secretase through the amyloidogenic pathway results in the generation of Amyloid beta $(A \beta)$, often linked with the pathological progression of the Alzheimer's disease (AD) [4]. AD is the most prevalent form of dementia in the elderly. It has been already shown that APP cleavage is impaired with inhibition of NCT function in vitro and in vivo [5]. It pinpoints to an indispensable role of NCT as a functional component of $\gamma$-secretase for regulated intramembrane proteolysis of APP. Emerging studies suggest that NCT plays a critical role in the assembly of the $\gamma$-secretase complex and in the docking of the substrate 
molecules to the catalytic zone $[5,6]$. NCT has also been identified as a substrate selector molecule that allows substrates that have shedded their ectodomain to be cleaved by the catalytic subunit of the $\gamma$-secretase. NCT is also known to interact with various proteoforms of APP and several other substrate molecules such as Notch [6-10]. It is already known that alterations in the balance of the proteoforms generated through the non-amyloidogenic and amyloidogenic pathway are crucial contributors to the development and progression of AD [11].

The spatial proximity of $\gamma$-secretase to its substrate either by local confinement within nanodomains or by random diffusional collisions on the plasma membrane is a requisite for generation of different proteoforms [12-15]. Due to its ability to regulate the APP proteolysis through the final biochemical step to produce $\mathrm{A} \beta, \mathrm{NCT}$ can modify the balance between the detrimental and non-detrimental proteoforms. NCT is also reported to regulate the synaptic function by modulating the short-term and long-term synaptic plasticity [16]. Additionally, AD is considered to begin with organizational deficits that contributes to molecular progression of disease pathology at individual synapses $[12,13,17]$. Despite the emerging evidence on the importance of NCT in regulating synaptic plasticity and in the pathogenesis of $\mathrm{AD}$, the nanoscale heterogeneity of $\mathrm{NCT}$ at synapses remains vague. Here, with the aid of ensemblebased nanoscopic imaging and analysis, we investigated the subsynaptic organization of NCT in functional zones of excitatory synapses and on the neuronal processes at nanoscale. Moreover, we also demonstrate the nanoorganization of NCT with APP and PS1, the catalytic subunit of $\gamma$-secretase, confirming the existence of their differential molecular aggregation at functional zones of the excitatory synapses. Additionally, we observed a subset of NCT domains independent of PS1, illustrating potentially unexplored regulatory mechanisms where the integral components of $\gamma$-secretase could work independent of each other.

\section{Results and discussion}

To comprehend the molecular distribution of NCT within different functional zones of an excitatory synapse, we evaluated the nanoscale spatial heterogeneity of NCT by comparing its association with a presynaptic marker for the cytomatrix at the active zone (CAZ), a postsynaptic marker for postsynaptic density (PSD) and a perisynaptic marker for the endocytic zone (EZ). The distribution profile of NCT within different functional zones of the synapse was assessed by multi-colour ensemble-based super resolution imaging using Stimulated Emission Depletion microscopy (STED) and Airyscan super resolution microscopy (Fig. 1 and Additional file 1: Fig.S1). Using Airyscan super resolution microscopy, we observed a similar localization profile of NCT when evaluated against different markers for CAZ, namely Bassoon and Piccolo as well as against different markers of PSD, namely Shank2 and PSD95 (Additional file 1: Fig. S1A-F). A detailed workflow for the segmentation paradigm used to perform morpho-functional characterization of functional zones of an excitatory synapse is summarized in Supplementary information (Additional file 1: Materials and methods). We then evaluated the nanoscopic association of NCT within functional zones of the synapse using Bassoon (CAZ), Shank2 (PSD) and Dynamin (EZ) as synaptic reference markers using STED microscopy. NCT molecular domains (nanodomains) namely, nanodomain ${ }_{\mathrm{NCT} / \mathrm{pre}}$, nanodomain $_{\mathrm{NCT} /}$ post and nanodomain ${ }_{\mathrm{NCT} / \text { peri }}$ indicate NCT nanodomains in regions marked positive for CAZ/PSD/EZ functional zones of the synapse (Fig. 1A-C). A gallery of representative STED images with synaptic markers and the associated nanodomains of NCT is presented (Fig. 1A-C). The morphological (length) and biophysical (intensity) traits of NCT nanodomains in pre/post/perisynapse were quantified (Fig. 1D, E). The distribution profile of length and intensity of nanodomain $\mathrm{NCT}_{\mathrm{N} \text { pre }}$, nanodomain $\mathrm{NCT}_{\mathrm{N} \text { post }}$

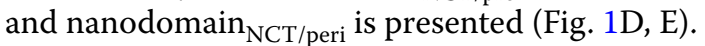

The length of nanodomain ${ }_{\mathrm{NCT} / \text { post }}$ was significantly lower compared to both nanodomain ${ }_{\mathrm{NCT}}$ / pre and nanodomain ${ }_{\mathrm{NCT} / \text { peri }}$. On the other hand, the length of nanodomain ${ }_{\mathrm{NCT} / \mathrm{pre}}$ was similar to that of nanodomain $_{\mathrm{NCT} / \text { peri }}$ (Additional file 1: Fig. S2A, B). The intensity of NCT nanodomains also showed a similar pattern with nanodomain $\mathrm{NCT}_{\mathrm{N} \text { post }}$ being significantly lower than that of nanodomain ${ }_{\mathrm{NCT} / \mathrm{pre}}$ and nanodomain NCT/ $_{\mathrm{N}}$ peri, while the intensity of nanodomain ${ }_{\mathrm{NCT} / \mathrm{pre}}$ was similar to that of nanodomain ${ }_{\mathrm{NCT} / \text { peri }}$ (Additional file 1: Fig.

(See figure on next page.)

Fig. 1 Subsynaptic compartmentalization of Nicastrin within functional zones of an excitatory synapse using STED microscopy. A-C Nanoscale distribution of Nicastrin (magenta) in pre/post/perisynapse with pseudocolour overlay of a presynaptic marker for the cytomatrix at the active zone (CAZ) in $\mathbf{A}$, a postsynaptic marker for postsynaptic density (PSD) in $\mathbf{B}$ and a perisynaptic marker for the endocytic zone (EZ) in $\mathbf{C}$ i.e., Bassoon, Shank2 and Dynamin (green), respectively. Overlap between functional zones of the synapse and Nicastrin is presented in black. $(1,2,3,4,5,6)$ are magnified insets of regions indicated in $\mathbf{A}-\mathbf{C}$. Scale bar in $\mathbf{A}-\mathbf{C}$ indicates $2.25 \mu \mathrm{m}$ (left) and $300 \mathrm{~nm}$ (right, inset). D, E Indicate the distribution of the length $\mathbf{D}$ and intensity $\mathbf{E}$ of Nicastrin nanodomains in pre/post/perisynaptic compartments. F, G Comparison of RSP $\mathbf{F}$ and RSE G for quantifying colocalization of Nicastrin for functional zones (pre/CAZ, post/PSD, peri/EZ) of an excitatory synapse. The data are represented as mean \pm SEM. Significance was determined by one-way analysis of variance (ANOVA) test followed by Tukey's multiple comparison test. Indications of significance correspond to $P$ values ${ }^{*} P \leq 0.05,{ }^{* *} P \leq 0.01$, and ${ }^{* *} P \leq 0.001$, ns $P>0.05 . n=5155$ (pre), 3016 (post) and 3966 puncta (peri) from 3-4 biological repeats 


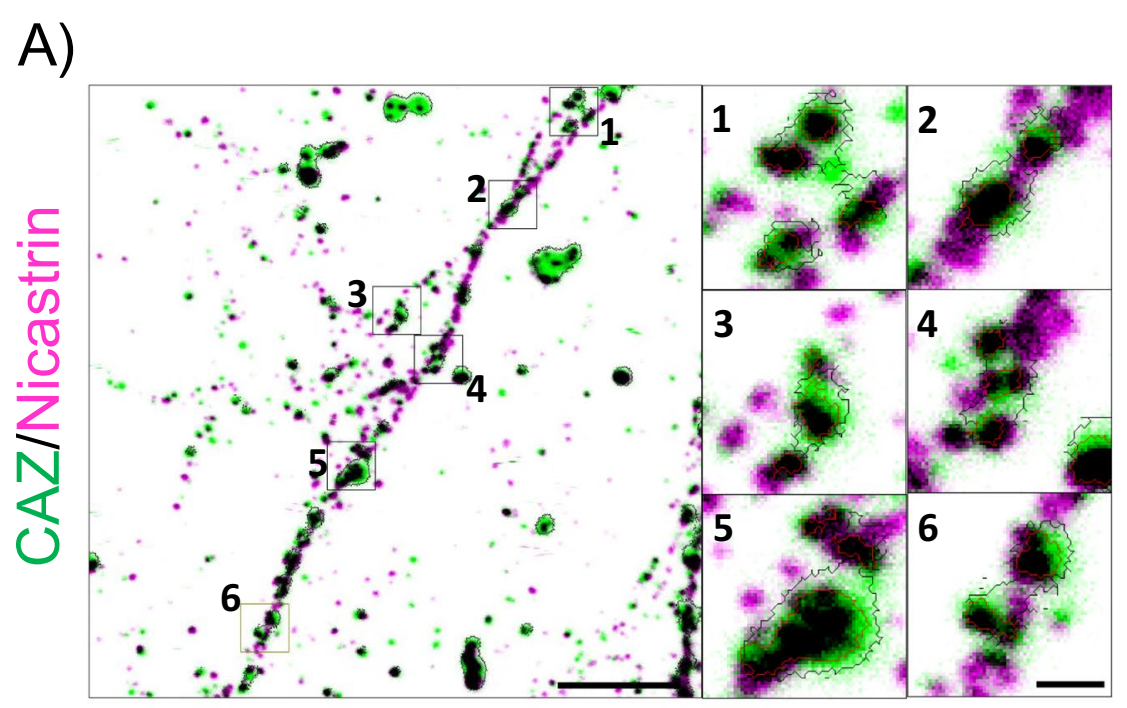

B)

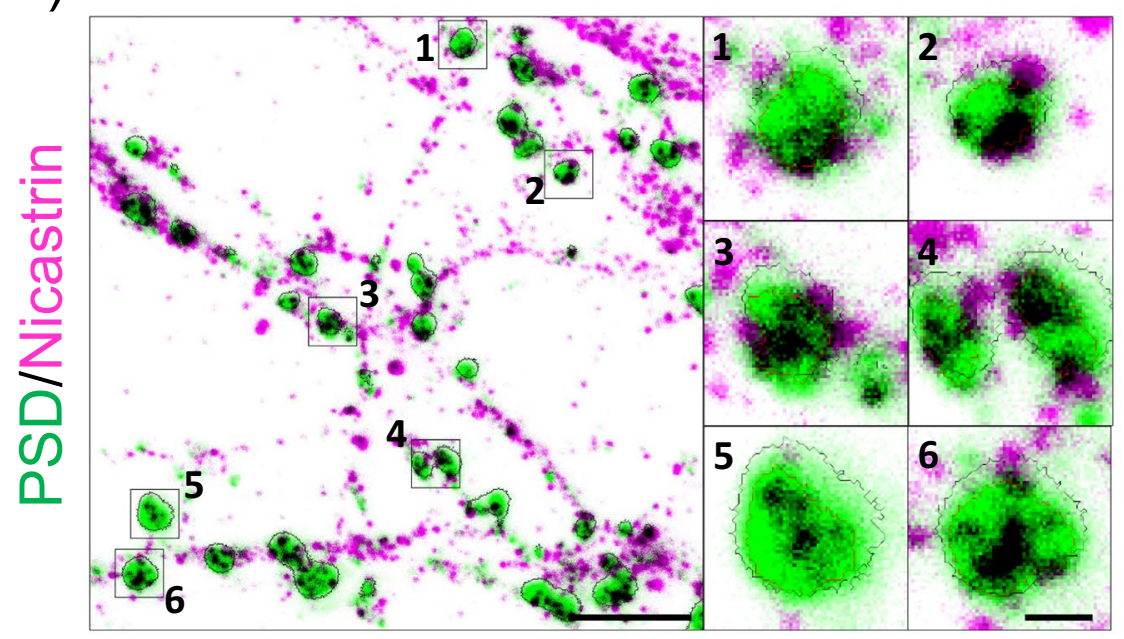

C)
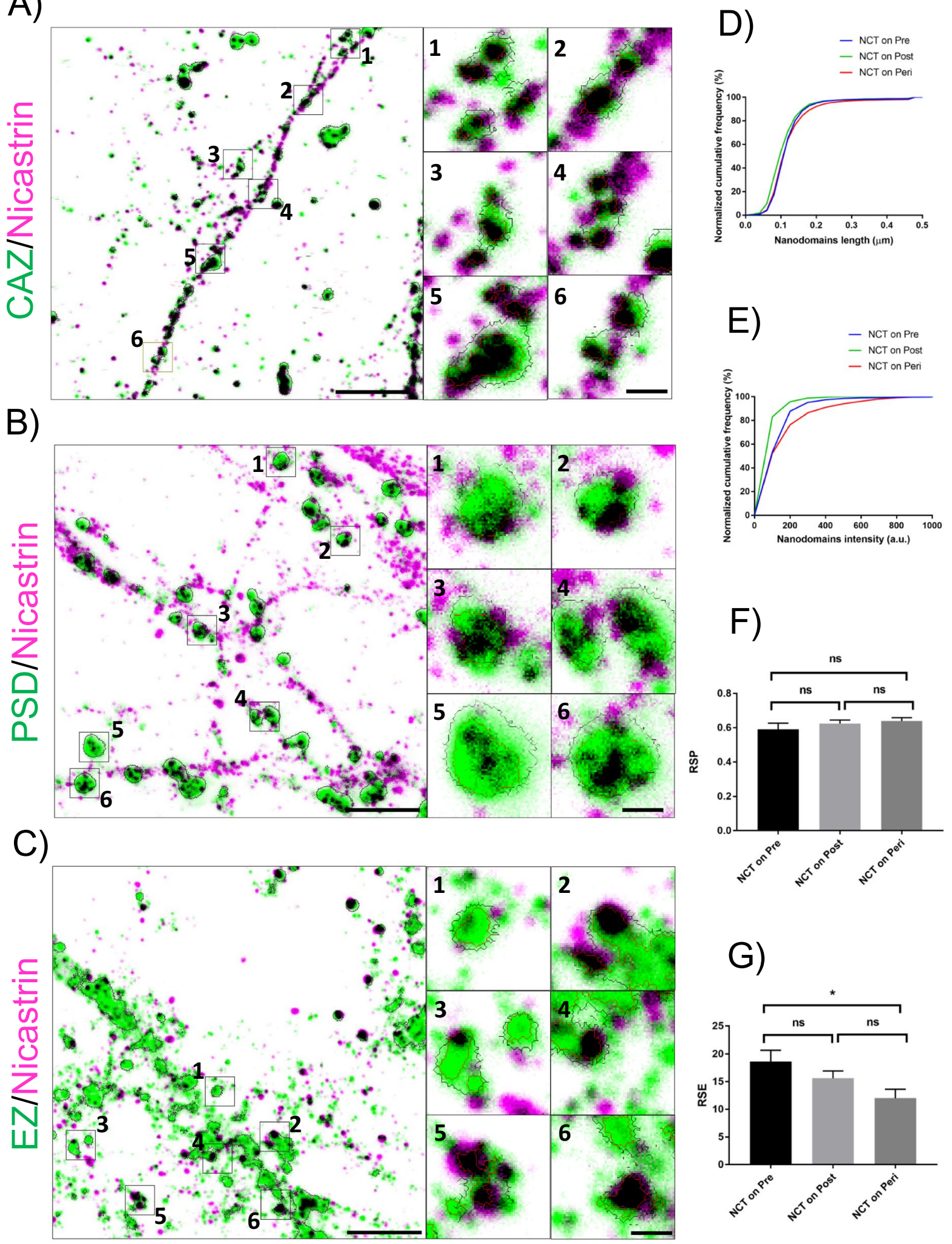

F)

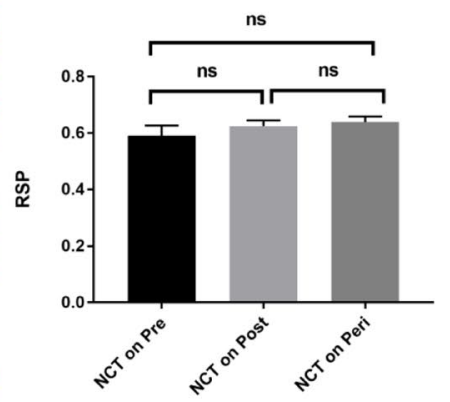

G)

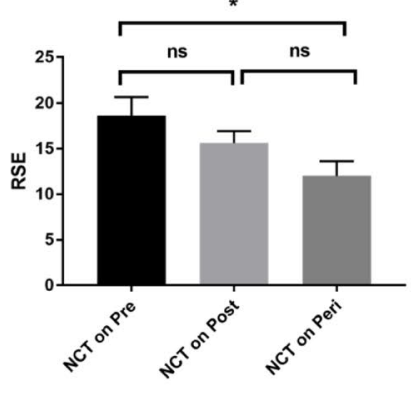

Fig. 1 (See legend on previous page.) 
S2A, B). The length and intensity of the nanodomains of NCT present in neuronal processes (Additional file 1: Fig. S2C, D) and in synaptic subcompartments (Fig. 1D, E and Additional file 1: Fig. S2A, B) are summarized in (Additional file 1: Table S1). Our observations confirm that NCT, which is an integral component of the $\gamma$-secretase, is distributed into nanoscale domains within sub-diffraction sized functional zones of the synapses. The morphological attributes of the NCT nanodomains are comparable to that of PS1 nanodomains reported previously [13]. Our observations thus confirm that $\gamma$-secretase is organized into nanodomains within neuronal sub compartments. Next, we found that the colocalization of NCT in CAZ/PSD/EZ did not differ significantly between each other when measured by average spatial overlap across regions using Resolution Scaled Pearson's (RSP) coefficient (Fig. 1F, G). On the contrary, the overall variability in fluorescence intensity across individual pixels in CAZ/PSD/EZ quantified by Resolution Scaled Error (RSE) between NCT on presynapse and perisynapse was significantly different, except when these zones were compared to the postsynapse (Fig. 1F, G). These observations of ensemble heterogeneity confirm the spatial variability of molecular organization of NCT, as revealed by nanodomain analysis. Additionally, NCT was also observed in inhibitory neurons (Additional file 1: Fig. S3A, B) and was associated with Gephyrin, a marker for inhibitory postsynapse on the hippocampal pyramidal neurons (Additional file 1: Fig. S3C).

The proteolytic processing of APP is deemed to occur by sequential cleavage, where the final snip is made by the $\gamma$-secretase [11]. Therefore, it was imperative to understand the nanoscopic association of NCT with APP as well as with the catalytic subunit, PS1. To quantify this association, we analyzed NCT regions marked positive for the presence of either APP or PS1. A gallery of STED images with PS1 and APP and the associated nanodomains of NCT is presented (Additional file 1: Fig. S4A, B). The nanodomain ${ }_{\mathrm{NCT}}$ associated with PS1 and APP are referred to as nanodomain ${ }_{\mathrm{NCT} /}$ PS and nanodomain ${ }_{\mathrm{NCT} / \mathrm{APP}}$, respectively. The distribution of length and intensity of nanodomain $\mathrm{NCT}_{\mathrm{NPS}}$ and nanodomain $_{\mathrm{NCT} / \mathrm{APP}}$ is shown (Additional file 1: Fig. S5A, B). Our results show a significantly higher length

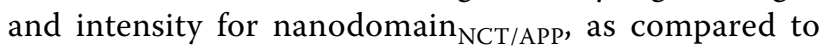
nanodomain $_{\mathrm{NCT} / \mathrm{PS}}$ (Additional file 1: Fig. S5C, D). Further, we found a significantly higher colocalization of NCT with PS1 compared to APP. In contrast, the variability of the quantified parameters of NCT with PS1 and with APP was similar (Additional file 1: Fig. S5E, F). In addition to the localization of NCT with PS1 indicating a functional $\gamma$-secretase, we also observed a subset of NCT nanodomains with variable intensities not localized to the proximity of PS1 (Additional file 1: Fig.S4A) and APP (Additional file 1: Fig. S4B). These observations are consistent with previous evidences on regulatory roles of NCT, independent of PS1 and $\gamma$-secretase complex [18]. It remains unclear if the individual components of $\gamma$-secretase could be transported independently to subcellular compartments, but it highlights novel unexplored mechanisms where these subunits could play an independent regulatory, anchoring or signaling role.

In the last decade, there has been a remarkable improvement in understanding the role of synaptic molecular organization in health and disease [12-15, 17, 19]. $\gamma$-secretase is involved in proteolysis of several substrate molecules and interact with molecules involved in synaptic transmission and plasticity [20]. We confirm that subunits of $\gamma$-secretase organize into discrete nanodomains on neuronal processes and within functional domains of excitatory synapses. Additionally, we observe that NCT and PS1 also form nanodomains independent of each other, where their functions could be independent of the $\gamma$-secretase activity. These observations of nanodomains of NCT and associated molecules in amyloidogenic processing highlight the role of multiprotein complexes in the proteolytic processing of APP. Our results are in resonance with the observation of the generation of $A \beta$ in megadalton sized protein complexes isolated biochemically [21]. Though we attribute the proteolysis of APP by secretases as diffusional collisions, there is very little understanding on how the molecular trapping of substrates and secretases can result in the differential generation of proteoforms. These observations provide insight on how the molecular organization of individual components of multiprotein complexes can modulate synaptic imbalance and heterogeneity, leading to spatial differences in the breakdown of APP and other enzyme substrates at individual excitatory synapses. In addition to its canonical role as a component of $\gamma$-secretases, NCT is also involved in the regulation of short- and long-term plasticity [16, 22], further confirming its role as a synaptic molecule whose localization, regulation and function needs to be explored in depth.

\section{Abbreviations}

PS1: Presenilin 1; NCT: Nicastrin; APH1: Anterior Pharynx Defective 1; PEN2: Presenilin Enhancer 2; APP: Amyloid Precursor Protein; A $\beta$ : Amyloid beta; AD: Alzheimer's Disease; CAZ: Cytomatrix at the Active Zone; PSD: Post Synaptic Density; EZ: Endocytic Zone; STED: Stimulated Emission Depletion microscopy. 


\section{Supplementary Information}

The online version contains supplementary material available at https://doi. org/10.1186/s13041-021-00855-x.

Additional file 1: Table S1: Summary of the quantitative estimation of morphological and biophysical properties of the different nanodomains of Nicastrin obtained using STED microscopy. Figure S1. Distribution of Nicastrin within functional zones of an excitatory synapse using Airyscan super resolution microscopy. A, C Indicate the pseudocolour coded distribution of the post- (Shank2 and PSD95) and pre-synaptic markers (Bassoon and Piccolo). B, D Indicate the pseudocolour coded distribution of Nicastrin and overlay with corresponding markers for functional zones of the synapse. The pseudocolour overlay of Nicastrin (green) with the postsynaptic marker Shank2 in red and the presynaptic marker Bassoon in blue is shown in $\mathbf{B}$. The pseudocolour overlay of Nicastrin (green) with the postsynaptic marker PSD95 in red and the presynaptic marker Piccolo in blue is shown in $\mathbf{D}$. E Magnified view of the boxed regions from pseudocolour overlay in $\mathbf{B}$ and $\mathbf{D}$. Scale bar in $\mathbf{B}, \mathbf{D}$ indicate $15 \mu \mathrm{m}$ and in $\mathbf{E} 2 \mu \mathrm{m}$. $\mathbf{F}$ Represents line scans connecting the centroids of pre- and post-synaptic reference molecules, indicating the distribution of Nicastrin with Shank2, Bassoon and PSD95, Piccolo. The X-and Y-axis represent the length $(\mu \mathrm{m})$ and normalized intensity (a.u.) respectively. Figure S2. Quantification of the nanoscale architecture of Nicastrin clusters within different functional zones of a synapse and on neuronal processes using STED microscopy. A, B Diversity in Nicastrin (median/IQR 25-75\% interval) clusters with respect to nanodomain length $\mathbf{A}$ and intensity $\mathbf{B}$ in pre/post/perisynapse. Significance was determined by Kruskal-Wallis test followed by Dunn's multiple comparison test. Indications of significance correspond to P values ${ }^{*} P \leq 0.05,{ }^{* *} P \leq 0.01$, and ${ }^{* * *} P \leq 0.001$, $n s P>0.05 . n=5155$ (pre), 3016 (post) and 3966 (peri) puncta from 3-4 biological repeats. C, D Indicate the nanoscale architecture of Nicastrin clusters on the neuronal processes. The distribution of the length of Nicastrin nanodomains is shown in $\mathbf{C}$ and the intensity in $\mathbf{D}$. $n=3521$ nanodomains from 3-4 biological repeats. Figure S3. Distribution of Nicastrin in inhibitory neurons and within inhibitory synapses of pyramidal neurons using confocal microscopy. A Evaluation of the presence of Nicastrin in Parvalbumin expressing GABAergic inhibitory neurons. The pseudocolour overlay represents Nicastrin in green and parvalbuminin red. The white arrows indicate the presence of both Nicastrin and parvalbumin, while the red arrows indicate the presence of Nicastrin in the absence of parvalbumin. Scale bar in A indicate $28 \mu \mathrm{m}$ (upper panel) and $11 \mu \mathrm{m}$ (lowerpanel). B Evaluation of the presence of Nicastrin in Parvalbumin/Calbindin expressing GABAergic inhibitory neurons. The pseudocolour overlay represents Nicastrin in green, parvalbumin in red and Calbindin in blue. Scale bar in B indicate $18 \mu \mathrm{m}$. C Indicate the distribution of Nicastrin within inhibitory synapses of pyramidal neurons. The pseudocolour overlay indicates Nicastrin ingreen and Gephyrin, a marker for the inhibitory postsynapses in magenta. The blue arrows indicate the clusters of Nicastrin overlapping with Gephyrin, while the yellow arrows indicate their independent distribution. Scale bar in C indicate $11 \mu \mathrm{m}$ (left) and $2 \mu \mathrm{m}$ (right, inset). Figure S4. Discrete nanoscale association of Nicastrin with PS1 and APP on neuronal processes using STED microscopy. A STED image of Nicastrin (magenta) with pseudocolour overlay of PS1 (green). (1, 2,3) are magnifiedinsets of regions indicated in A. B STED image of Nicastrin (magenta) withpseudocolour overlay of APP (green). $(1,2,3)$ are magnified insets of regions indicated in $\mathbf{B}$. Scale bar in $\mathbf{A}$, B indicates $3 \mu \mathrm{m}$ (left) and $750 \mathrm{~nm}$ (right).The black contours represent segmented regions marking the continuous stretc.hof neuronal processes, marking the presence of Nicastrin. Figure

S5. Quantification of the nanoscale architecture of Nicastrin clusters associating with PS1 and APP on neuronal processes using STED microscopy. A, B Indicate the distribution of the length $\mathbf{A}$ and intensity B of Nicastrin nanodomains with PS1 and APP. C, D Diversity in nanodomain length $\mathbf{C}$ and intensity D of Nicastrin clusters (median/IQR25-75\% interval) associating with PS1 and APP. Significance was determined by unpaired two-tailed Mann-Whitney test. E, F Comparison of RSP $\mathbf{E}$ and RSE F for quantifying colocalization of Nicastrin with respect to PS1 and APP. The data are represented as mean \pm SEM. Significance was determined by two-tailed unpaired Student's t-test with Welch's correction. Indications of significance correspond to $P$ values ${ }^{*} P \leq 0.05$, ${ }^{* *} P \leq 0.01$, and ${ }^{* * *} P \leq 0.001$, ns $P>0.05 . n=5417$ (Nicastrin on PS1) and 7163 (Nicastrinon APP) puncta from 3-4 biological repeats. Figure S6. Identification of synapse associated endocytic zone. A, B The workflow to detect synapse associated Dynamin (endocytic zones) using a combination of both super resolution (STED) and conventional (confocal) imaging paradigms. A The confocal image of PSD marker and STED image of Dynamin were selected and thresholded to detect regions with high molecular content. They were then size filtered and obtained regions were converted into binary images. B The overlay of the masks confirms the overlap of segmented clusters of Dynamin with PSD. The automated evaluation of synaptic masks in the Dynamin positive domains was performed. Absence of Dynamin in PSD positive regions (green regions) were considered as negative, presence of both markers as positive (red regions) and dynamin alone as false positive (magenta regions). The paradigm involves sequential segmentation protocols to isolate Dynamin that is associated with the synaptic compartments and marking these segmented regions as synaptic endocytic zones. Scale bar in A, B indicate $5 \mu \mathrm{m}$. (Please refer to Supplementary information (Additional file 1: Materials and methods))

Figure S7. Identification of Dynamin associated with Clathrin.

A Pseudocolour overlay of super resolved images of Clathrin (green) associated with Dynamin (Magenta) obtained using STED microscopy. B The workflow on super resolution images (STED) to detect Dynamin clusters colocalized with Clathrin, another marker for endocytic zone. The STED image of endocytic markers namely, Clathrin and Dynamin were selected and thresholded to detect regions with high molecular content. They were then size filtered and obtained regions were converted into binary images. The overlay of the masks confirms overlap of segmented clusters of Clathrin with Dynamin. Automated evaluation of Clathrin in the Dynamin positive domains was performed. For this purpose, the automatically detected Dynamin positive regions were transferred to the binary masks positive for Clathrin. Absence of Clathrin signal in Dynamin positive region was considered as negative, and those regions were not considered as endocytic regions. This segmentation protocol selectively evaluates domains enriched in both Clathrin and Dynamin, and therefore the presence of functional endocytic machinery. Scale bar in $\mathbf{A}$ indicate $5 \mu \mathrm{m}$ and in $\mathbf{B} 2.25 \mu \mathrm{m}$. (Please Refer to Supplementary information (Additional file 1: Materials and methods)).

\section{Acknowledgements}

The authors thank the Bioimaging facility at IISc and NCBS, Bangalore, India. We also thank the Central Animal Facility at IISc, Bangalore, India. We thank Dr. Jean Baptiste Sibarita, IINS, Bordeaux for sharing novel analysis tools. We are thankful to Ms. Vihashini Selvakumar for technical assistance.

\section{Authors' contributions}

SK and DN designed research. SK, KM, MJ and DN performed all the experiments unless otherwise indicated. SK, KM and DN performed analysis. PRN and MJ prepared neuronal cell cultures. SSS shared critical reagents. SK, MJ and DN wrote the manuscript. All the authors read, provided critical inputs, and approved the final version of the manuscript.

\section{Funding}

This work was supported by generous grants from Department of Biotechnology (Innovative Young Biotechnologist Award to DN and MJ), Department of Biotechnology Genomics Engineering Taskforce to DN, DBT Ramalingaswami Fellowship to DN and MJ, DBT-IISC Partnership program to DN, IISC-STAR program grant to DN, Science and Engineering Research Board (Early Career Research Award to MJ), Indian Institute of Science (Institute of Excellence Program) and University Grants Commission, India to DN and Tata Trusts, India for the program grant (co-investigator-DN). SK thank postdoctoral fellowship support through Department of Biotechnology, Genomics Engineering Taskforce grant awarded to DN. PRN acknowledge graduate fellowship support from ICMR (New Delhi, India).

\section{Availability of data and materials}

The datasets collected and analysed during the current study is available from the corresponding author on reasonable request. 


\section{Declarations}

Ethics approval and consent to participate

All experiments involving animals were carried out in accordance with institutional guidelines for the use and care of animals after approval from the Institutional Animal Ethics Committee (IAEC), Indian Institute of Science, Bangalore, India.

\section{Consent for publication}

Not applicable.

\section{Competing interests}

The authors declare that they have no competing interests.

\section{Author details}

${ }^{1}$ Centre for Neuroscience, Indian Institute of Science, 560012 Bangalore, India.

${ }^{2}$ Centre for Molecular Neurobiology, Department of Neurobiology, The University of Chicago, 60637 Chicago, IL, USA.

Received: 9 June 2021 Accepted: 9 September 2021

Published online: 13 October 2021

\section{References}

1. De Strooper B. Aph-1, Pen-2, and Nicastrin with Presenilin generate an active $\gamma$-Secretase complex. Neuron. 2003;38:9-12.

2. Gertsik N, Chiu D, Li YM. Complex regulation of $y$-secretase: from obligatory to modulatory subunits. Front Aging Neurosci. 2015;7:342.

3. Li T, Ma G, Cai H, Price DL, Wong PC. Nicastrin is required for assembly of presenilin/Y-secretase complexes to mediate notch signaling and for processing and trafficking of $\beta$-amyloid precursor protein in mammals. J Neurosci. 2003;23:3272-7.

4. Sisodia SS, St George-Hyslop PH. $Y$-Secretase, notch, A $\beta$ and alzheimer's disease: where do the presenilins fit in? Nat Rev Neurosci. 2002:3:281-90.

5. Verdile G, Gandy SE, Martins RN. The role of presenilin and its interacting proteins in the biogenesis of Alzheimer's beta amyloid. Neurochem Res. 2007;32:609-23.

6. Elad N, Strooper B, De, Lismont S, Hagen W, Veugelen S, Arimon M. The dynamic conformational landscape of gamma-secretase. J Cell Sci. 2015;128:589-98.

7. Shah S, Lee SF, Tabuchi K, Hao YH, Yu C, LaPlant Q, et al. Nicastrin functions as a $\gamma$-secretase-substrate receptor. Cell. 2005;122:435-47.

8. Bolduc DM, Montagna DR, Gu Y, Selkoe DJ, Wolfe MS. Nicastrin functions to sterically hinder $\gamma$-secretase-substrate interactions driven by substrate transmembrane domain. Proc Natl Acad Sci U S A. 2016:113:E509-18.
9. Hu C, Zeng L, Li T, Meyer MA, Cui MZ, Xu X. Nicastrin is required for amyloid precursor protein (APP) but not Notch processing, while anterior pharynx-defective 1 is dispensable for processing of both APP and Notch J Neurochem. 2016;136:1246-58.

10. Petit D, Hitzenberger M, Lismont S, Zoltowska KM, Ryan NS, Mercken $M$, et al. Extracellular interface between APP and Nicastrin regulates A length and response to $\gamma$-secretase modulators. EMBO J. 2019;38:1-18.

11. Selkoe DJ, Hardy J. The amyloid hypothesis of alzheimer's disease at 25 years. EMBO Mol Med. 2016:8:595-608.

12. Kedia S, Ramakrishna P, Netrakanti PR, Jose M, Sibarita JB, Nadkarni S, et al. Real-time nanoscale organization of amyloid precursor protein. Nanoscale. 2020;12:8200-15.

13. Kedia S, Ramakrishna P, Netrakanti PR, Singh N, Sisodia SS, Jose M, et al. Alteration in synaptic nanoscale organization dictates amyloidogenic processing in Alzheimer's disease. iscience. 2021;24:101924.

14. Schedin-Weiss S, Caesar I, Winblad B, Blom H, Tjernberg LO. Superresolution microscopy reveals $\gamma$-secretase at both sides of the neuronal synapse. Acta Neuropathol Commun. 2016:4:29.

15. Escamilla-Ayala AA, Sannerud R, Mondin M, Poersch K, Vermeire W, Paparelli $L$, et al. Super-resolution microscopy reveals majorly mono-and dimeric presenilin1/Y-secretase at the cell surface. Elife. 2020;9:1-30.

16. Lee SH, Sharma M, Südhof TC, Shen J. Synaptic function of nicastrin in hippocampal neurons. Proc Natl Acad Sci U S A. 2014;111:8973-8.

17. Kedia S, Nair D. Nanoscale rearrangement of APP organization as a therapeutic target for Alzheimer's disease. Med Hypotheses. 2020;143:110143.

18. Pardossi-Piquard R, Dunys J, Giaime E, Guillot-Sestier MV, St. George-Hyslop P, Checler F, et al. P53-Dependent control of cell death by nicastrin: Lack of requirement for presenilin-dependent $\gamma$-secretase complex. J Neurochem. 2009;109:225-37.

19. Zhang H, Zhang C, Vincent J, Zala D, Benstaali C, Sainlos M, et al. Modulation of AMPA receptor surface diffusion restores hippocampal plasticity and memory in Huntington's disease models. Nat Commun. 2018;9:4272.

20. Jurisch-yaksi N, Sannerud R, Annaert W. A fast growing spectrum of biological functions of $\gamma$-secretase in development and disease. Biochim Biophys Acta. 2013;1828:2815-27.

21. Liu L, Ding L, Rovere M, Wolfe MS, Selkoe DJ. A cellular complex of BACE1 and $\gamma$-secretase sequentially generates $A \beta$ from its full-length precursor. J Cell Biol. 2019;218:644-63.

22. Tabuchi K, Chen G, Südhof TC, Shen J. Conditional forebrain inactivation of nicastrin causes progressive memory impairment and age-related neurodegeneration. J Neurosci. 2009;29:7290-301.

\section{Publisher's Note}

Springer Nature remains neutral with regard to jurisdictional claims in published maps and institutional affiliations.
Ready to submit your research? Choose BMC and benefit from:

- fast, convenient online submission

- thorough peer review by experienced researchers in your field

- rapid publication on acceptance

- support for research data, including large and complex data types

- gold Open Access which fosters wider collaboration and increased citations

- maximum visibility for your research: over $100 \mathrm{M}$ website views per year

At BMC, research is always in progress.

Learn more biomedcentral.com/submissions 